\title{
Caracterización de la cultura organizacional de los establecimientos comerciales del Centro Comercial San Pedro Plaza de la ciudad de Neiva
}

\section{Characterization of the organizational culture of the commercial facilities of Centro Comercial San Pedro Plaza in the city of Neiva}

\author{
Carlos Javier Martínez Moncaleano \\ Administrador de empresas. \\ Cartan1991@hotmail.com \\ Sofía Medina Cárdenas \\ Estudiante de administración de Empresas. \\ Universidad Surcolombiana. Neiva. \\ Sofiamedina19@hotmail.com
}

\section{Resumen}

El presente documento sintetiza del desarrollo y los resultados de la investigación "Caracterización de la Cultura Organizacional de los Establecimientos Comerciales del Centro Comercial San Pedro Plaza de la Ciudad de Neiva" adelantado como proyecto del Semillero de Investigación "Se Busca", de la Facultad de Economía y Administración de la Universidad Surcolombiana.

Se parte del complejidad de las organizaciones y del rol de la cultura organizacional en la concepción de las mismas, seguidamente se expone el diseño metodológico de la investigación y el trabajo de campo que se realizó para su elaboración por medio de las técnicas de encuestas, entrevistas y observación directa. Finalmente se muestran las conclusiones y resultados el que se destaca que, si bien, en los locales del centro existe una cultura organizacional fuerte, hay algunos factores que deben ser mejorados.

Palabras clave: Cultura Organizacional, Creencias, Valores, Actitudes, Satisfacción.

\begin{abstract}
This document synthesizes the development and the results of the research "Characterization of the Organizational Culture of the Commercial Facilities of Centro Comercial San Pedro Plaza of the City of Neiva" carried out as a project of "Se Busca" School of Research from the School of Economics and Administration of Universidad Surcolombiana.

Starting from the complexity of organizations and the role of organizational culture in the conception of them, following is the presentation of the methodological design of the research and of the field work that was done for its elaboration by means of the techniques of surveys, interviews, and direct observation. Finally, the conclusions and results are shown in which it is highlighted that, although there is a strong organizational culture in the premises of the shopping center, there are some factors that must be improved.
\end{abstract}

Key Words: Organizational culture, beliefs, values, attitudes, satisfaction.

\section{Introducción}

La concepción de las organizaciones ha sufrido profundos e importantes cambios desde la formulación de los modelos de administración científica y clásica expuestos por autores como Frederick Taylor, Henry Ford o Henri Fayol. En primer lugar, hay que considerar las escuelas y los modelos que se han formulado en el último siglo y que han configurado diversos paradigmas administrativos y organizacionales, así como los constantes cambios sociales, culturales, políticos y económicos tales como fueron el gigantismo industrial, -etapa que se forjó entre las dos guerras mundiales y que marcó los cimientos de la aldea globalizada-, la caída del bloque comunista, -lo que supuso el fin de la guerra fría-, el auge económico de los países asiáticos, el predominio del modelo neoliberal por sobre el keynesiano y el marxista en las economías mundiales o los inmensos avances en el campo de la comunicación y de la informática que se han vivenciado en los últimos años, todo esto ha hecho que las organizaciones se configuren como entes complejos, cada vez más difíciles de predecir y que vivan sumergidas en el mar de incertidumbre del macro entorno y de su concepción interna. 
Partiendo de esto y considerando que las organizaciones son sistemas abiertos $y$, ante todo, entes colectivos nacidos debido básicamente por razones sociales (interacciones humanas), razones materiales y por efecto sinérgico (Hicks, Gullet, 1971) en las que existe una importante interacción con el entorno externo y que tienen a nivel interno una complejidad y una mutabilidad similar a la de su macro entorno, se puede afirmar que no existe una organización sin una cultura inherente e intrínseca que identifique, oriente y distinga su accionar estratégico, así como su imagen como colectividad en el mercado (Agudelo y Velázquez, 2011) por ello lograr caracterizar la cultura de una organización, es importante por cuanto permite su mejoramiento continuo, así como una correcta formulación estratégica en las mismas.

Por tanto, se plantea entonces que el presente proyecto de investigación surgió a raíz de determinar las características de la cultura organizacional del Centro Comercial San Pedro Plaza de la ciudad de Neiva que, desde su irrupción en el mercado regional en el año 2005 , se ha convertido en un punto neurálgico en el comercio del sur del país, así como en un gestor de nuevos e innovadores modelos de negocio en la ciudad aportando indiscutiblemente al crecimiento económico y empresarial de la región.

Por ello, el objetivo principal del presente estudio fue:

- Determinar las características de la cultura organizacional, en términos de valores, creencias y actitudes, de los miembros que conforman los establecimientos comerciales del Centro Comercial San Pedro Plaza de la ciudad de Neiva.

Además, los objetivos específicos que se plantearon fueron:

- Identificar el tipo de cultura organizacional presente en estas empresas.

- Determinar las características de la cultura gerencial de los gerentes de los establecimientos comerciales.

- Identificar como los clientes de San Pedro Plaza perciben la cultura organizacional de sus establecimientos comerciales en relación al servicio dispensado.

\section{Marco Referencial}

Ante todo se puede afirmar que la cultura organizacional ha adquirido gran importancia al interior de las organizaciones, por lo que ha sido centro de numerosas investigaciones. Los enfoques sobre cultura organizacional han abarcado diferentes manifestaciones culturales: valores, creencias, ritos, historias, etc., hasta el rol del gerente como emisor de la cultura de las organizaciones. (Cujár, Ramos, Hernández, Riaño y López, 2011), en consecuencia, el estudio de la cultura organizacional es fundamental tanto en las ciencias administrativas, como en la sociología organizacional y en la psicología industrial; hay que considerar que la cultura organizacional es el espejo de la compañía y que en ella se reflejan sus principios, valores, actitudes, mentalidad, estilo de vida, normas, comportamientos, reglas, filosofía y clima organizacional. Una empresa debe tener bien definida su cultura, y dentro de ella preocuparse por la satisfacción de todas las partes interesadas, para así elaborar y aplicar correctamente sus estrategias, administrar y utilizar adecuadamente la información (Cantillo, Álzate, Galindo, Arteta, Ladines, Serje, 2011), por tanto el análisis la caracterización y el fortalecimiento de la cultura de una empresa es un punto neurálgico de su concepción estratégica y un factor central en su funcionamiento y desarrollo.

Tomando en consideración lo anterior hay que, en primer lugar, tener en cuenta algunas definiciones aceptadas en el ámbito académico sobre este tópico. Para Ouchi (1982), por ejemplo, la cultura organizacional es una serie de símbolos, ceremonias y mitos que comunican al personal de las empresas los valores y las creencias más arraigados dentro de la organización, en cambio Chiavenato (2001) afirma que la cultura organizacional reposa sobre un sistema de valores, tradiciones, creencias y actitudes, como forma aceptada y estable en las relaciones sociales de cada organización, asimismo, Shein (2007) considera que el termino debería reservarse nivel más profundo de presunciones básicas y creencias que comparten los miembros de una empresa.

Dentro de los modelos más aceptados de la tipología de cultura organizacional se encuentra el de Robbins (2004), cuyo modelo clasifica las culturas de débiles o de fuertes, en su modelo las culturas fuertes se caracterizan porque los valores de la organización se aceptan con firmeza y son ampliamente compartidos, caso contrario a las culturas débiles en el que una sólo una minoría comparte los valores de la organización. 
Figura 1. Características de la cultura Organizacional Débil y la Cultura organizacional Fuerte

\begin{tabular}{|lll|}
\hline \multicolumn{1}{|c|}{ CARACTERISTICAS } & \multicolumn{1}{c|}{$\begin{array}{c}\text { CULTURA DÉBIL } \\
\text { AUTONOMIA INDIVIDUAL }\end{array}$} & $\begin{array}{l}\text { Cupervisión estrecha } \\
\text { Cupervisión general }\end{array}$ \\
\hline ESTRUCTURA & Puestos de trabajo estandarizados & Puestos de trabajo flexibles \\
\hline APOYO & $\begin{array}{l}\text { Enfoque de la gerencia a la } \\
\text { producción }\end{array}$ & Enfoque de la gerencia al personal \\
\hline RECOMPENSA & $\begin{array}{l}\text { Se desconocen los niveles } \\
\text { productivos del personal }\end{array}$ & $\begin{array}{l}\text { Compensaciones y asensos basados } \\
\text { en niveles de productividad }\end{array}$ \\
\hline $\begin{array}{l}\text { TOLERANCIA AL } \\
\text { CONFLICTO }\end{array}$ & $\begin{array}{l}\text { Poca, Escaso o nulo conflicto } \\
\text { constructivo }\end{array}$ & $\begin{array}{l}\text { Poco conflicto destructivo, Gerencia } \\
\text { apoya el Conflicto funcional o } \\
\text { constructivo }\end{array}$ \\
\hline VALORES & Valores son aceptados con firmeza. & $\begin{array}{l}\text { Valores son asimilados de forma } \\
\text { insuficiente. }\end{array}$ \\
& & \\
\hline
\end{tabular}

\section{Metodología}

El estudio fue de tipo básico con enfoque descriptivo y perceptivo y de naturaleza tanto cuantitativa como cualitativa, aplicado directamente al campo de los hechos debido a sus propósitos exploratorios. Su diseño fue no experimental y de corte transversal. La población de estudio estuvo constituida por los miembros de los 250 locales comerciales del Centro Comercial San Pedro Plaza, así como por usuarios del centro. Las principales variables contempladas en la investigación son: cultura organizacional, cultura gerencial, creencias, valores, actitudes, clima laboral y satisfacción del cliente.

Para la recolección de información de las fuentes primarias implicadas en la realización del estudio se utilizaron encuestas de selección múltiple aplicadas a los clientes del centro comercial, entrevistas de profundidad hechas a los gerentes de los locales y observación directa, y las fuentes secundarias están conformadas por la teleología del centro comercial y sus políticas de calidad.

El trabajo de campo del proyecto, en el que se recolectaron los datos se realizó entre el 15 de abril del 2015 y el 1 de julio del 2015. Durante estas fechas se hicieron las entrevistas, se gestionaron las encuestas y se hizo uso de la técnica de observación directa dentro del centro comercial, para posteriormente realizar análisis de resultados y concluir el estudio. En lo que respecta a las entrevistas de profundidad, fueron consultados diez gerentes de establecimientos comerciales además de la señora Cielo Ortiz, administradora del centro comercial. Para ello se hizo una programación previa de cada una de las entrevistas en las que se dejaron las pautas de las mismas y los temas a tratar. Las entrevistas se realizaron dentro de los establecimientos y tuvieron una duración promedio de siete minutos, salvo a la realizada a Cielo Ortiz la cual se prolongó por veinte minutos. Las entrevistas arrojaron luces sobre los valores, la teleología, los modelos gerenciales y el clima laboral interno que se presenta en el centro y sus establecimientos.

La encuesta fue aplicada a 100 usuarios con base a un modelo de muestreo aleatorio simple con un margen de error del $7 \%$ y un nivel de confianza del $95 \%$ para poblaciones infinitas. La encuesta está conformada por 9 enunciados o ítems, los cuales, fueron calificados por los individuos de la muestra en una escala de Likert; una vez diligenciadas todas las encuestas se hizo un profundo análisis estadístico a cada uno de los ítems de la encuesta; se usaron técnicas de estadística descriptiva como análisis gráfico por medio de gráficos de torta y Box Plot, medidas de tendencia central y de dispersión y análisis correlacional. Esto permitió determinar las percepciones del cliente con respecto a la cultura organizacional presente en los establecimientos y su percepción de los principales valores, creencias y actitudes de los locales. Asimismo se hizo observación directa en los establecimientos del centro comercial San Pedro plaza como método complementario.

\section{Resultados}

Una vez realizado el trabajo de campo del estudio y habiéndose hecho un análisis tanto cuantitativo como cualitativo de los datos recolectados, se determinó que en primer lugar, la presentación personal de los trabajadores, así como la estética y la limpieza de los establecimientos comerciales, son valores fundamentales en la configuración de estas empresas y que gran parte del atractivo que encuentran los clientes al visitar el centro comercial se debe a la armonía visual que este posee, además, que la gerencia ejerce un importante esfuerzo para llegar a este cometido. De igual manera, se estableció que los miembros que conforman los establecimientos comerciales del centro tienen un gran sentido de pertenencia por los mismos y por tanto mantienen una actitud favorable 
en sus funciones laborales. Además, un punto importante y a tener en cuenta es que existe poco rumor mal intencionado en los establecimientos y la opinión de los trabajadores sobre sus puestos de trabajo es generalmente positiva lo que, como es lógico pensar, se configura como algo propicio en la concepción de la cultura organizacional de los mismos, esto quedó claro tanto en el desarrollo de las entrevistas de profundidad como en la observación directa que se ejerció durante el estudio.

Un punto importante y que implica una ventaja competitiva en los establecimientos parte de que se presenta una cultura gerencial dada a ambientes democráticos y participativos. Pese a haber líneas jerárquicas bien definidas, en los establecimientos se manejan modelos de comunicación participativos, además de notarse relaciones de compañerismo entre los jefes y los subordinados, lo que permite establecer que existe la creencia de que, si los trabajadores tienen cierto grado de empoderamiento en la empresa y son tratados de manera asertiva se van a lograr mejores resultados y los objetivos van a ser alcanzados con una mayor facilidad. Por otro lado, en el centro comercial hay una clara actitud hacia el servicio, es decir que se realiza un esfuerzo conjunto en busca de ofrecer al cliente un servicio integral en elementos que involucran etapas de pre compra, compra y pos compra. El análisis hecho a las entrevistas de profundidad permite vislumbrar que la disciplina se configura otro valor fundamental en los locales comerciales y en su concepción de mercado.

Un punto no tan favorable y que marca un factor a considerar, radica en el hecho de que existe cierta disonancia entre cómo es percibido el servicio dispensado por los establecimientos comerciales entre los gerentes y los clientes. Mientras los gerentes consideran que valores como honestidad, amabilidad y calidad del servicio son los valores más importantes y determinantes dentro de la concepción de sus empresas, los clientes creen que, pese a que existen estos valores dentro de los establecimientos, no son tan categóricos, adicionalmente de que en ocasiones el servicio no es lo suficientemente ágil y oportuno. Se presentan ciertos inconvenientes con respecto a la información de productos y cumplimiento de garantías, además, la tasa de servicio (el número de clientes atendidos a una razón de tiempo) nos es del todo satisfactoria en algunos casos.

\section{Conclusiones}

Los establecimientos comerciales de San Pedro Plaza muestran una cultura organizacional fuerte, en el que es innegable el énfasis de la gerencia por una actitud hacia el mejoramiento y la consolidación de valores y principios basada en la creencia de que el cliente es el centro de la organización y en el que valores como el orden, la disciplina o la solidaridad son fundamentales en su cultura como empresas, además, se puede afirmar que en términos generales, los usuarios están satisfechos con los servicios del centro comercial y todo lo que este representa para la ciudad y la región. Sin embargo, hay que considerar que aspectos como la agilidad en el servicio podrían ser mejores, esto en miras de un mayor fortalecimiento organizacional, por tanto es factible implementar un estudio sobre tiempos de espera el cual permita calcular aspectos tales como el tiempo promedio de espera de los usuarios la hora de realizar su compra o la media de usuarios haciendo cola para ser atendidos. Lo que podría incrementar la agilidad en los servicios ofrecidos y lograr enaltecer la creencia que prima entre los miembros del centro comercial, de que el cliente es el centro y la razón de ser de sus empresas y que siempre se debe velar por su satisfacción y bienestar.

\section{Bibliografía}

Agudelo B., Velázquez L., (2011). Descripción de la cultura organizacional en una institución educativa. Tesis de maestría no publicada. Universidad Tecnológica de Pereira. Pereira. Colombia

Cantillo E., Alzate A., Galindo K., Arteta A., Landinez D., Serje N. (2011). Influencia de la Cultura Organizacional en la Competitividad de las Empresas. Medellín: Ninth LACCEI

Chiavenato I., (2001). Introducción a la Teoría General de la Administración. México D.C: Mcgraw-Hill

Cújar A., Ramos C., Hernández H., López J. (2011). Cultura organizacional: evolución en la medición. Santiago de Cali: ICESI

Oushi W. (1985). LaTeoria Z: Cómo Pueden Los Empresas Hacer Frente Al Desafío Japones. Barcelona: Ediciones Orbis, S.A

Shein E. (2009). Corporate Culture survival Guide. Washington D.C: Library of Congress Cataloging-in-Publication Data

Hicks H. y Gulley R. (1971). The Management of Organizations and Human Resources Approach. Washington D.C: Mcgraw-Hill

Robbins S., (2004). Comportamiento Organizacional. México D.C: Pearson 